\title{
Serum lactate level and mortality in metformin-associated lactic acidosis requiring renal replacement therapy: a systematic review of case reports and case series
}

Hung-Chieh Yeh${ }^{1}$, I-Wen Ting ${ }^{1}$, Ching-Wei Tsai ${ }^{1,2}$, Jenn-Yu Wu ${ }^{3}$ and Chin-Chi Kuo ${ }^{1,2^{*}}$

\begin{abstract}
Background: The current practice concerning timing, mode, and dose of renal replacement therapy (RRT) in patients with metformin-associated lactic acidosis (MALA) with renal failure remains unknown. To investigate whether serum lactate level and prescription pattern of RRT are associated with mortality in patients with MALA requiring RRT.

Methods: We searched PubMed/Medline and EMBASE from inception to Sep 2014 and applied predetermined exclusion criteria. Case-level data including case's demographics and clinical information related to MALA were abstracted. Multiple logistic regression modeling was used to examine the predictors of mortality.

Results: A total of 253 unique cases were identified with cumulative mortality of 17.2\%. Eighty-seven percent of patients had acute kidney injury. Serum lactate level was significantly higher in non-survivors (median $22.5 \mathrm{mmol} / \mathrm{L}$ ) than in survivors $(17.0 \mathrm{mmol} / \mathrm{L}, p$-value $<0.01)$ and so did the median blood metformin concentrations $(58.5 \mathrm{vs} .43 .9 \mathrm{mg} / \mathrm{L}$, $p$-value $=0.05$ ). The survival advantage was not significantly different between the modalities of RRT. The adjusted odds ratio of mortality for every one $\mathrm{mmol} / \mathrm{L}$ increase in serum lactate level was $1.09(95 \% \mathrm{Cl} 1.02-1.17, p$-value $=0.01)$. The dose-response curve indicated a lactate threshold greater than $20 \mathrm{mmol} / \mathrm{L}$ was significantly associated with mortality.

Conclusions: Our study suggests that predialysis level of serum lactate level is an important marker of mortality in MALA patients requiring RRT with a linear dose-response relationship. To better evaluate the optimal prescription of RRT in MALA, we recommend fostering an international consortium to support prospective research and large-scale standardized case collection.
\end{abstract}

Keywords: Metformin, Acute kidney injury, Metabolic acidosis, Renal replacement therapy, Lactate

\section{Background}

Metformin is recommended as the first-line pharmacological agent for type 2 diabetes by international guidelines for its weight-neutrality and strong evidencebased cost-effectiveness in preventing diabetic vasculopathy $[1,2]$. The spectrum of clinical application of

\footnotetext{
* Correspondence: chinchik@gmail.com

${ }^{1}$ Kidney Institute and Division of Nephrology, Department of Internal Medicine, China Medical University Hospital and College of Medicine, China Medical University, 2, Yude Rd., North Dist, Taichung City 404, Taiwan

${ }^{2}$ Big Data Center, China Medical University Hospital and College of Medicine, China Medical University, Taichung, Taiwan

Full list of author information is available at the end of the article
}

metformin has continued to widen and presently include metabolic syndrome and polycystic ovarian syndrome [3, 4]. The most feared adverse effect of metformin use is lactic acidosis due to the historically reported mortality of approximately $50 \%$ during the period of $1960-2000$ and about $25 \%$ since 2000 [5]. Although metformin-associated lactic acidosis (MALA) is rare, with a stable estimated incidence of 1 to 10 per 100,000 [6-8], the stigma concern greatly limit the use of metformin in elderly populations where heart failure and renal impairment are common conditions [9]. 
The rarity and fatality of MALA make it difficult to obtain conclusive evidence regarding predisposing and prognostic factors as most studies performed to date have been retrospective single-center or registry-based ecological studies with small sample sizes and without blood metformin levels. Consequently, whether to discontinue metformin in patients with cardiac or renal dysfunction remains undetermined and a growing body of evidence demands a critical re-evaluation of contraindications for metformin [7]. More importantly, standard management of MALA is uncertain albeit renal replacement therapy (RRT) has been considered crucial in treating severe form of MALA for its ability to provide renal support, eliminate metformin, and optimize acidbase status [7]. The role of timing, dose, and mode of RRT in MALA is also unknown. To understand the current practice and trend of RRT in MALA, we systematically reviewed all case reports of MALA requiring RRT in the literature worldwide. As the selection of RRT mode is highly institutionalized and inherent spectrum bias in a single-center study, a systematic review of all case reports would be more informative to describe the RRT prescription pattern in MALA. We also evaluated the epidemiological and clinical prognostic factors that associate with mortality of MALA complicated by at least renal failure.

\section{Methods}

\section{Search strategy and study selection}

The systematic search and review processes were conducted in accordance with the Preferred Reporting Items for Systematic Reviews and Meta-Analyses (PRISMA) Statement criteria [10]. We searched PubMed/Medline and EMBASE for case reports of metformin-associated lactic acidosis (MALA) requiring renal replacement therapy by combining three key research concepts derived from relevant $\mathrm{MeSH}$ and text-word terms such as "renal replacement therapy", "dialysis", "metformin", and "lactic acidosis". Additional file 1: Table S1 showed the full search strategy. The search period was January 1966 through Sep 15, 2014. There were no language restrictions. We also manually reviewed the reference lists from relevant review articles and the investigators' files.

Our exclusion criteria were: 1) reviews, case series, editorials, and research letters that did not provide individual biochemical data; 2) when metformin was not the only offending factor causing lactic acidosis; 3 ) cases that did not received renal replacement therapy in the disease course; 4) phenformin- or buformin-related lactic acidosis cases; 5) when survival status of an individual patient could not be ascertained. Duplicate publication between conference abstracts and full papers was identified by authors' group and the demographics of the cases (Additional file 1: Figure S1).

\section{Data abstraction}

Two authors, C.C. Kuo and H.C. Yeh, independently abstracted data from the articles that met the selection criteria. We developed a data extraction form to record the characteristics of the study (authors, journal, years of publication, and country); the characteristics of participants (basic demographics, underlying medical conditions, and outcome data on mortality or end stage renal disease); initial presentations (symptoms and signs); information on biochemical indicators of MALA (peak serum creatinine level, peak blood lactate level, and blood gas measurements); mode and dose of renal replacement therapy (RRT); the causes of death (solely related to MALA or not). In the event of disagreement, other nephrologists, C.W. Tsai and I.W. Ting, were consulted and a consensus was reached.

\section{Definition of key variables}

All information in this review including baseline demographics was directly extracted from the primary literature at individual level. The diagnostic criteria for MALA described in this review refers to an arterial $\mathrm{pH}$ less than 7.35 and a serum lactate level above $5 \mathrm{mmol} / \mathrm{L}$ in association with metformin exposure, or based on the author's clinical diagnosis where papers not providing measurements of either $\mathrm{pH}$ or lactate level [11]. The baseline renal function was defined as the lowest serum creatinine concentration that could be traced back prior to the index event. Peak serum creatinine and lactate levels were defined as the highest serum creatinine and lactate concentrations, respectively, after the index event and before RRT initiation (Additional file 1: Figure S2). Other clinical parameters related to MALA including $\mathrm{pH}$, serum bicarbonate, and glucose were also abstracted using the reported values closest in time prior to the peak lactate level or the same time. Values greater than the highest bound and less than the lowest bound of the individual's laboratory reportable range were assigned the highest laboratory reportable value and a half of the detection limit, respectively. Cases with post-MALA ESRD were defined as being long-term RRT dependent after the index event. Cases with acute kidney injury (AKI) was defined either by the authors of the source paper or by an absolute decline from baseline renal function. Inappropriateness of metformin dosing was defined by (1) the contraindicated use of metformin if serum creatinine higher than 1.4 and $1.5 \mathrm{mg} / \mathrm{dL}$ in women and men, respectively, based on the US-FDA black-box warning and (2) the prescribed dose higher than the maximal dosage recommended in a recently proposed dosing algorithm based on the CKD stage $[12,13]$.

\section{Modalities of renal replacement therapy}

Based on the detailed description of these published reports, RRT modalities were classified into four main 
categories: (1) Peritoneal dialysis (PD); (2) Intermittent renal replacement therapy (IRRT) including intermittent hemodialysis (IHD), bicarbonate hemodialysis, and hemodialysis with hemoperfusion; (3) Prolonged intermittent RRT (PIRRT) including prolonged hemodialysis and sustained low efficiency dialysis (SLED); (4) Continuous renal replacement therapy (CRRT) including continuous venovenous hemofiltration $(\mathrm{CVVH})$, continuous venovenous hemodialysis (CVVHD), hemofiltration (HF), continuous venovenous hemodiafiltration (CVVHDF), and high volume hemofiltration (HVHF). The intensification of RRT was defined as a modality transition from IRRT to PIRRT or CRRT, or a higher dialysis dose delivery during the same RRT modality.

\section{Other variables}

Metformin usage was categorized into three groups: regular, suicidal and accidental. The daily and total metformin dosage was recorded, respectively, in patients with regular metformin use and suicidal or accidental ingestion. Medications that may impair kidney function and predispose the development of MALA, including nonsteroidal anti-inflammatory drugs (NSAIDs), angiotensin-converting-enzyme inhibitors (ACEIs), angiotensin II receptor blockers (ARBs), diuretics, aspirin, and statins were abstracted. Both baseline and trough estimated glomerular filtration rates (eGFR) were calculated by using the CKD Epidemiology Collaboration (CKD-EPI) equation [14].

\section{Statistical methods}

Continuous variables were expressed as median with interquartile range and categorical variables were presented as frequencies and percentages. The KolmogorovSmirnov test was used together with histograms to assess the normality of continuous data. To examine the time trend of acute renal care, we stratified the study data before and after 2005 as the concepts of chronic kidney disease and acute kidney injury has rapidly evolved in the past 10 years $[15,16]$. Statistical differences between parametric continuous variable were estimated by Wilcoxon signed rank test whereas differences between categorical variables were evaluated with Chi-square test.

The relationships between serum lactate concentration and serum metformin concentration and between the estimated metformin ingestion dose and serum metformin concentration were described using both parametric and nonparametric methods. Multiple logistic regression modeling was used to examine the predictors of mortality. The proposed adjustment variables included blood lactate, age, sex, RRT modality, metformin usage, and peak creatinine level. We also conducted several sensitivity analyses including (1) restricting the analyses only to cases whose metformin levels were available, (2) additional adjustment for baseline and trough eGFR, or (3) additional adjustment for appropriateness of metformin dosing (Inapproriateness vs. appropriateness) in the multiple logistic model shown in Table 3. The results were consistent (data not shown).

Two-tailed values of $P<0.05$ were considered significant. Statistical analysis was performed using Stata SE Version 12 (StataCorp, College Station, Texas, USA) and R, version 3.0.2 (R Foundation for Statistical Computing, Vienna, Austria [www.r-project.org]). The map figure (Additional file 1: Figure S3) was drawn with the support of the googleVis library [17].

\section{Results}

\section{Clinical characteristics of selected cases}

For years 1977-2014, 142 case reports and 3 case series from 30 countries were identified with a total of 253 unique cases (Additional file 1: Table S2 and Figure S3) and $95.7 \%$ met the conventional diagnostic criteria of MALA. The cumulative mortality was $16.2 \%$ (41 deaths). There was no statistical difference in age at event, diabetes status, the use of metformin (including the estimated dosage), and the use of predisposing medication and other anti-diabetic agents between survival and non-survival groups (Table 1). Both groups presented with similar symptoms and signs. The level of consciousness was the most frequently reported $(79.8 \%$ of all selected cases) but only $56.9 \%$ of them had acute conscious change. The least frequently recorded vital sign was body temperature $(36.8 \%)$, yet $63.4 \%$ of them were classified as hypothermia $(<35.5 \mathrm{C}$ degree or defined by authors of the primary study) (Table 1). We found higher frequency of respiratory failure requiring endotracheal intubation (83.3\%) in non-survival group than in survival group $(55.1 \%)$ ( $p$-value $<0.01)$.

\section{Kidney response to MALA}

Nearly $5.4 \%$ of the selected cases had end stage-renal disease requiring renal replacement therapy before the event whereas $94.6 \%$ presented with acute kidney injury (Table 1). Baseline serum creatinine level were comparable between survivors and non-survivors; however, survivors had median peaked creatinine level two times higher than non-survivors $(7.2$ vs. $3.2 \mathrm{mg} / \mathrm{dL}, p$-value $<0.01$ ). Regarding metabolic markers of lactic acidosis, serum lactate level was significantly higher in nonsurvivors (median $22.0 \mathrm{mmol} / \mathrm{L}$ ) than in survivors $(17.0 \mathrm{mmol} / \mathrm{L}, p$-value $<0.01)$ (Table 1$)$. We found no statistical difference in $\mathrm{pH}$, blood metformin, and bicarbonate levels between the two groups. The distributions of renal replacement modalities, attempts of treatment intensification, and treatment duration between the survival and non-survival groups were no different 
Table 1 Characteristics of published cases between 1977 and 2014 by survival status ${ }^{c}$

\begin{tabular}{|c|c|c|c|c|}
\hline Variable & $\begin{array}{l}\text { Total } \\
\text { [N, proportion of total study } \\
\text { population, } 253 \text { patients] }\end{array}$ & $\begin{array}{l}\text { Alive } \\
\text { [n, proportion of total } \mathrm{N}] \\
212 \text { patients }\end{array}$ & $\begin{array}{l}\text { Deceased } \\
\text { [n, proportion of total N] } \\
41 \text { patients }\end{array}$ & $p$-value \\
\hline \multicolumn{5}{|l|}{ Demographics } \\
\hline Age (yr) & $64(52.5-72.5)[252,99.6 \%]$ & $64(54-72.5)[212,84.1 \%]$ & $60.5(42.5-73)[40,15.9 \%]$ & 0.40 \\
\hline Sex (male) & $41.8 \%[249,98.4 \%]$ & $38.5 \%[208,83.5 \%]$ & $58.5 \%[41,16.5 \%]$ & 0.02 \\
\hline Diabetes & $91.3 \%[231,96.9 \%]$ & $91.0 \%[210,83.0 \%]$ & $93.0 \%[43,17.0 \%]$ & 0.76 \\
\hline \multicolumn{5}{|l|}{ Medical history } \\
\hline Metformin use & {$[232,91.7 \%]$} & {$[196,84.5 \%]$} & {$[36,15.5 \%]$} & 0.11 \\
\hline Regular medication & $76.7 \%$ & $79.1 \%$ & $63.9 \%$ & \\
\hline Suicidal & $22.8 \%$ & $20.4 \%$ & $36.1 \%$ & \\
\hline Accidental & $0.4 \%$ & $0.5 \%$ & $0.0 \%$ & \\
\hline Estimated metformin dosage at MALA $(\mathrm{g})$ & $2.6(1.7-5.1)[181,72.0 \%]$ & $2.6(1.7-4.0)[154,85.0 \%]$ & $2.6(1.0-24.5)[27,14.9 \%]$ & 0.79 \\
\hline Predisposing medication ${ }^{\mathrm{b}}$ & $97.7 \%[83,32.8 \%]$ & $97.4 \%[76,91.6 \%]$ & $100 \%[7,8.2 \%]$ & 0.67 \\
\hline Other anti-diabetic agents & $95.7 \%[47,18.6 \%]$ & $97.5 \%[40,85.1 \%]$ & $85.7 \%[7,14.9 \%]$ & 0.15 \\
\hline \multicolumn{5}{|l|}{ Appropriateness of metformin use } \\
\hline Creatinine criteria & $28.8 \%[80,31.6 \%]$ & $25.0 \%[68,85 \%]$ & $50.0 \%[12,15 \%]$ & 0.08 \\
\hline eGFR-dosing criteria & $57.5 \%[73,28.9 \%]$ & $59.0 \%[61,83.6 \%]$ & $50.0 \%[12,16.4 \%]$ & 0.56 \\
\hline \multicolumn{5}{|l|}{ Symptoms \& Signs } \\
\hline Hypothermia & $63.4 \%[93,36.8 \%]$ & $64.5 \%[76,81.7 \%]$ & $58.8 \%[17,18.3 \%]$ & 0.66 \\
\hline Hypotension & $50.0 \%[162,64.0 \%]$ & $49.3 \%[136,84.0 \%]$ & $53.9 \%[26,16.1 \%]$ & 0.67 \\
\hline Abdominal pain & $35.6 \%[149,59.0 \%]$ & $37.3 \%$ [134. 89.9\%] & $20.0 \%[15,10.1 \%]$ & 0.18 \\
\hline Upper Gl discomfort & $77.4 \%[155,61.3 \%]$ & $78.4 \%[139,90.0 \%]$ & $68.8 \%[16,10.3 \%]$ & 0.38 \\
\hline Lower Gl discomfort & $43.7 \%[151,59.7 \%]$ & $44.1 \%[136,90.1 \%]$ & $40.0 \%[15,9.9 \%]$ & 0.76 \\
\hline Conscious disturbance & $56.9 \%[202,79.8 \%]$ & $56.3 \%[174,86.1 \%]$ & $60.7 \%[28,13.9 \%]$ & 0.66 \\
\hline \multicolumn{5}{|l|}{ Severity } \\
\hline Respiratory failure & $60.1 \%[168,66.4 \%]$ & $55.1 \%[138,82.1 \%]$ & $83.3 \%[30,17.9 \%]$ & $<0.01$ \\
\hline Kidney Injury Profile & {$[203,80.2 \%]$} & {$[175,86.2 \%]$} & {$[28,13.8 \%]$} & 0.66 \\
\hline Acute kidney injury & $94.6 \%$ & $94.9 \%$ & $92.9 \%$ & \\
\hline ESRD & $5.4 \%$ & $5.1 \%$ & $7.1 \%$ & \\
\hline \multicolumn{5}{|l|}{ Biochemical profile } \\
\hline Baseline creatinine $(\mathrm{mg} / \mathrm{dL})$ & $1.2(1.0-1.5)[104,41.1 \%]$ & $1.2(0.9-1.5)[88,84.6 \%]$ & $1.3(1.1-1.8)[16,15.4 \%]$ & 0.26 \\
\hline Baseline eGFR (ml/min/1.73m²) & $50.0(38.7-71.5)[102,40.3 \%]$ & $52.0(39.6-71.5)[86,84.3 \%]$ & $43.5(37.4-73.4)[16,15.7 \%]$ & 0.56 \\
\hline Peak creatinine (mg/dL) & 7.0 (3.3-9.5) [211, 83.4\%] & $7.2(4.2-9.7)[183,86.7 \%]$ & $3.2(1.8-7.2)[28,13.3 \%]$ & $<0.01$ \\
\hline Trough eGFR $\left(\mathrm{ml} / \mathrm{min} / 1.73 \mathrm{~m}^{2}\right)$ & $6.1(4.5-16.4)[207,81.8 \%]$ & $5.6(4.3-11.2)[179,86.4 \%]$ & $19.2(6.1-27.4)[28,13.5 \%]$ & $<0.01$ \\
\hline Metformin (mg/L) & $45(27.0-74.4)[85,33.6 \%]$ & 43.9 (27.0-71.0) [71, 83.5\%] & $58(16-150)[14,16.5 \%]$ & 0.61 \\
\hline Lactate $(\mathrm{mmol} / \mathrm{L})$ & $18.0(12.7-23)[234,92.5 \%]$ & $17(12.5-21.2)[198,84.6 \%]$ & $22(14.7-28.4)[36,15.4 \%]$ & $<0.01$ \\
\hline Bicarbonate $(\mathrm{mmol} / \mathrm{L})$ & $5.0(3.1-8.0)[170,67.1 \%]$ & $5.0(3.0-8.0)[146,85.9 \%]$ & $5.6(4.2-8.5)[24,14.1 \%]$ & 0.19 \\
\hline $\mathrm{pH}$ & $6.9(6.8-7.1)[241,95.3 \%]$ & $6.9(6.8-7.1)[203,84.2 \%]$ & $6.9(6.8-7.1)[38,15.8 \%]$ & 0.56 \\
\hline Glucose (mg/dL) & $120.9(36-22[118,46.6 \%]$ & $105.4(34.3-219.5)[105,88.1 \%]$ & $198.5(72-270)[14,11.9 \%]$ & 0.22 \\
\hline MALA criteria & $95.7 \%[253,100 \%]$ & $96.2 \%[212,83.8 \%]$ & $92.7 \%[41,16.2 \%]$ & 0.31 \\
\hline \multicolumn{5}{|l|}{ Renal replacement therapy (RRT) profile } \\
\hline Type of RRT & {$[251,99.2 \%]$} & {$[210,83.7 \%]$} & {$[41,16.3 \%]$} & 0.03 \\
\hline IRRT & $33.9 \%$ & $36.7 \%$ & $19.5 \%$ & \\
\hline PIRRT & $13.2 \%$ & $13.3 \%$ & $12.2 \%$ & \\
\hline
\end{tabular}


Table 1 Characteristics of published cases between 1977 and 2014 by survival status ${ }^{c}$ (Continued)

\begin{tabular}{|c|c|c|c|c|}
\hline CRRT & $50.6 \%$ & $48.6 \%$ & $61.0 \%$ & \\
\hline PD & $2.3 \%$ & $1.4 \%$ & $7.3 \%$ & \\
\hline RRT dose intensification & $11.2 \%[214,84.6 \%]$ & $9.8 \%[184,86.0 \%]$ & $20.0 \%[30,14.0 \%]$ & 0.10 \\
\hline RRT duration (day) & $2.0(1.0-3.0)[162,64.0 \%]$ & $2.0(1.0-4.0)[140,86.4 \%]$ & $1.5(1.0-2.0)[22,13.6 \%]$ & 0.11 \\
\hline Post-event ESRD & $3.9 \%[156,61.7 \%]$ & $3.3 \%[154,98.7 \%]$ & $50.0 \%[2,1.3 \%]$ & $<0.01$ \\
\hline
\end{tabular}

Abbreviations: CRRT continuous renal replacement therapy, eGFR estimated glomerular filtration rate, IRRT intermittent renal replacement therapy, MALA metformin-associated lactic acidosis, PD peritoneal dialysis, PIRRT prolonged intermittent renal replacement therapy

${ }^{a}$ Daily metformin dosage ( $\mathrm{g} /$ day) for patient on regular metformin; total dosage $(\mathrm{g})$ for patients with suicidal or accidental metformin exposure

${ }^{\mathrm{b}}$ Medication that may predispose the development of MALA included nonsteroidal anti-inflammatory drugs, angiotensin-converting-enzyme inhibitors, angiotensin II receptor blockers, diuretics, aspirin, and statins

${ }^{\circ}$ Characteristics of MALA patients are given as percentage in each categorical variable (e.g., male) or median (interquartile range) (e.g., age) in each continuous variable; $p$-values for categorical and continuous variables are derived from Pearson Chi-squared and Wilcoxon signed rank test, respectively

(Table 1). After 1993, PD was no longer reported as the RRT for patients with MALA who required renal support (Fig. 1).

\section{Appropriateness of metformin use}

Among patients receiving regular metformin dose, the proportion of inappropriate prescription of metformin was $28.9 \%$ and $57.5 \%$ based on FDA warning and a recently proposed eGFR-dosing algorithm, respectively (Table 2) [12, 13]. Patients with inappropriate metformin dosing had significantly lower baseline renal function but trough eGFR was comparable between the two groups regardless the classification criteria. When using the criteria according to FDA warning, patients with inappropriate metformin prescription were more likely to experience mortality and received a lower dose of metformin with a concomitantly lower serum lactate level compared to those of patients with appropriate dosing (Table 2). When using the eGFR-dosing approach, patients with inappropriate prescription were found to receive a significant higher dose of metformin (2550 mg vs. $1700 \mathrm{mg}, p$-value < 0.01) than the appropriate dosing group. However, there was no statistical difference in mortality proportion, serum metformin concentration, and serum lactate level between the two groups (Table 2).

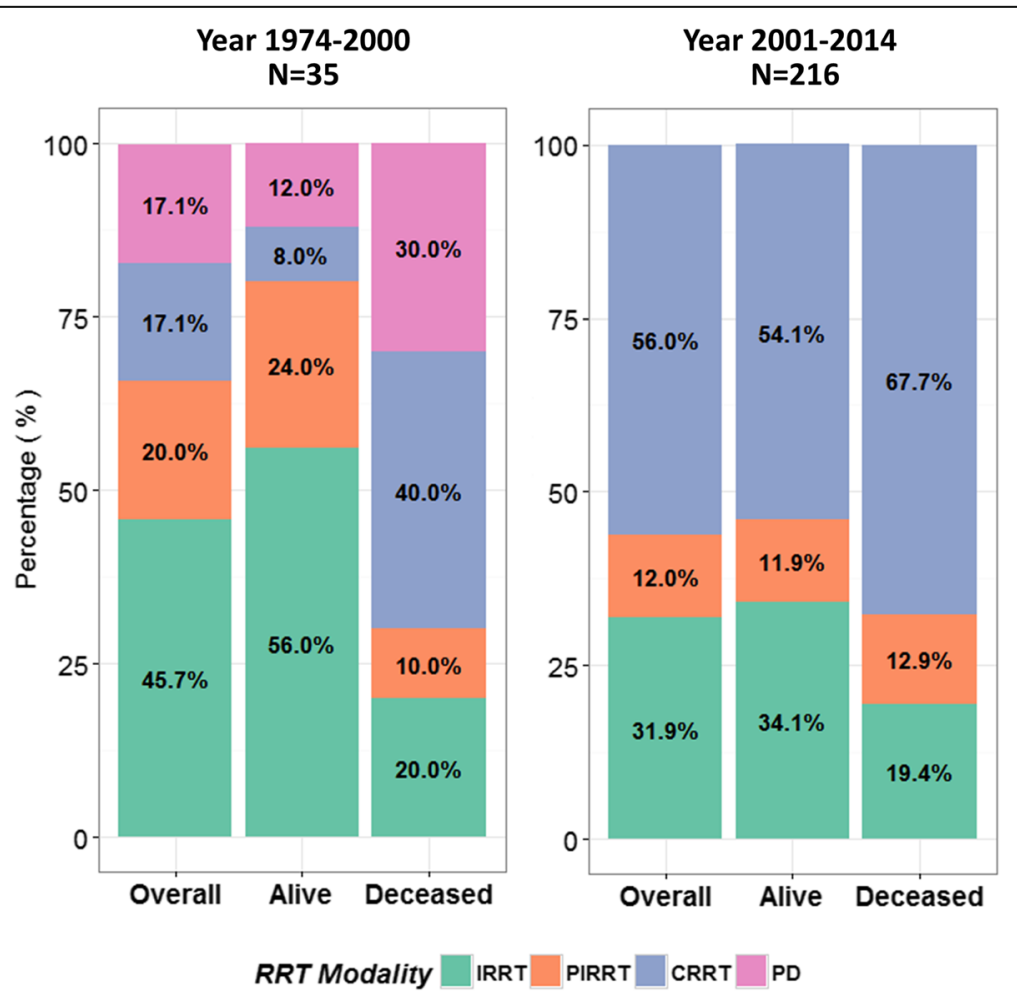

Fig. 1 The distribution of modality of renal replacement therapy (RRT) of the enrolled cases published from 1974 to 2000 and from 2001 to 2014 by survival status. Abbreviations: CRRT, continuous renal replacement therapy; IRRT, intermittent renal replacement therapy; PD, peritoneal dialysis; PIRRT, prolonged intermittent renal replacement therapy; RRT, renal replacement therapy 
Table 2 Clinical characteristics of published cases with available baseline serum creatinine and metformin dosage data stratified by dosing appropriateness ${ }^{a}$

\begin{tabular}{|c|c|c|c|}
\hline & Appropriate dosing & Inappropriate dosing & $p$-value \\
\hline \multicolumn{4}{|l|}{ FDA black-box warning } \\
\hline$n$ & 57 & 23 & \\
\hline Age (yr) & $67(60-74)$ & $73(63-76)$ & 0.28 \\
\hline Male (Frequency, \%) & $20(35.1)$ & $9(39.1)$ & 0.73 \\
\hline Baseline eGFR (ml/min/1.73m²) & $58.1(46.5-71.5)$ & $31.2(18.8-35.3)$ & $<0.01$ \\
\hline \multicolumn{4}{|l|}{ Baseline CKD stage } \\
\hline CKD stage 1-2 (Frequency, \%) & $26(45.6)$ & $0(0)$ & $<0.01$ \\
\hline CKD stage 3 (Frequency, \%) & $31(54.4)$ & $13(56.5)$ & \\
\hline CKD stage 4-5 (Frequency, \%) & $0(0)$ & $10(43.5)$ & \\
\hline Trough eGFR (ml/min/1.73m²) & $\begin{array}{l}n=56 \\
5.5(4.3-8.7)\end{array}$ & $\begin{array}{l}n=21 \\
6.7(4.5-14.8)\end{array}$ & 0.66 \\
\hline Mortality (Frequency, \%) & $6(10.5 \%)$ & $6(26.1 \%)$ & 0.08 \\
\hline Metformin dose (mg/day) & $2000(1500-3000)$ & $1700(1500-2550)$ & 0.04 \\
\hline Metformin concentration (mg/L) & $\begin{array}{l}n=29 \\
37(22-52)\end{array}$ & $\begin{array}{l}n=11 \\
26(16-31.5)\end{array}$ & 0.21 \\
\hline Lactate (mmol/L) & $19(12.5-23.5)$ & $13.4(9.2-16.6)$ & 0.02 \\
\hline \multicolumn{4}{|l|}{ eGFR-dosing algorithm } \\
\hline$n$ & 31 & 42 & \\
\hline Age $(y r)$ & $67(60-73)$ & $70.5(63-76)$ & 0.46 \\
\hline Male (Frequency, \%) & $16(51.6)$ & $11(26.2)$ & 0.03 \\
\hline Baseline eGFR (ml/min/1.73m²) & $65.7(51.3-78.1)$ & $38.2(31.1-46.4)$ & $<0.01$ \\
\hline \multicolumn{4}{|l|}{ Baseline CKD stage } \\
\hline CKD stage 1-2 (Frequency, \%) & $20(64.5)$ & $3(7.1)$ & $<0.01$ \\
\hline CKD stage 3 (Frequency, \%) & $11(35.5)$ & $29(69.1)$ & \\
\hline CKD stage 4-5 (Frequency, \%) & $0(0)$ & $10(23.8)$ & \\
\hline Trough eGFR (ml/min/1.73m²) & $\begin{array}{l}n=31 \\
5.7(4.5-20.5)\end{array}$ & $\begin{array}{l}n=40 \\
5.5(3.8-10.3)\end{array}$ & 0.22 \\
\hline Mortality (Frequency, \%) & $6(19.4 \%)$ & $6(14.3 \%)$ & 0.56 \\
\hline Metformin dose (mg/day) & $1700(1500-2000)$ & $2550(1700-3000)$ & $<0.01$ \\
\hline Metformin concentration (mg/L) & $\begin{array}{l}n=16 \\
41.5(19-62.5)\end{array}$ & $\begin{array}{l}n=19 \\
27(20-43.9)\end{array}$ & 0.27 \\
\hline Lactate (mmol/L) & $\begin{array}{l}n=30 \\
17.5(11.8-24)\end{array}$ & $\begin{array}{l}n=41 \\
14.2(11.7-21)\end{array}$ & 0.27 \\
\hline
\end{tabular}

Abbreviations: CKD chronic kidney disease, eGFR estimated glomerular filtration rate, FDA Food and Drug Administration

${ }^{a}$ Characteristics are given as percentage in each categorical variable (e.g., male) or median (interquartile range) (e.g., age) in each continuous variable; $p$-values for categorical and continuous variables are derived from Pearson Chi-squared and Wilcoxon signed rank test, respectively

\section{Association among serum lactate level, blood metformin concentration, and mortality}

Serum lactate level was positively correlated with blood metformin concentration (Fig. 2a). Blood metformin concentration was also positively related to the metformin dose (Fig. 2b). For each increase in one unit of serum lactate, there was an associated 9\% (95\% CI, 2-17, $p$-value $=0.01$ ) increase in MALA-related mortality in multiple logistic regression. Factors associated with lower mortality were female gender and peaked serum creatinine level (Table 3). In the dose-response analysis, we found a linear association between serum lactate level and MALA-related mortality, particularly in the range of greater than $20 \mathrm{mmol} / \mathrm{L}$ (Fig. 3).

\section{Discussion}

This systematic review suggests that the cumulative mortality of MALA with kidney failure in case reports or case series published between 1977 and 2014 with biochemical data available at individual level is $17.2 \%$, which is much lower than previously documented high mortality rates to 
a

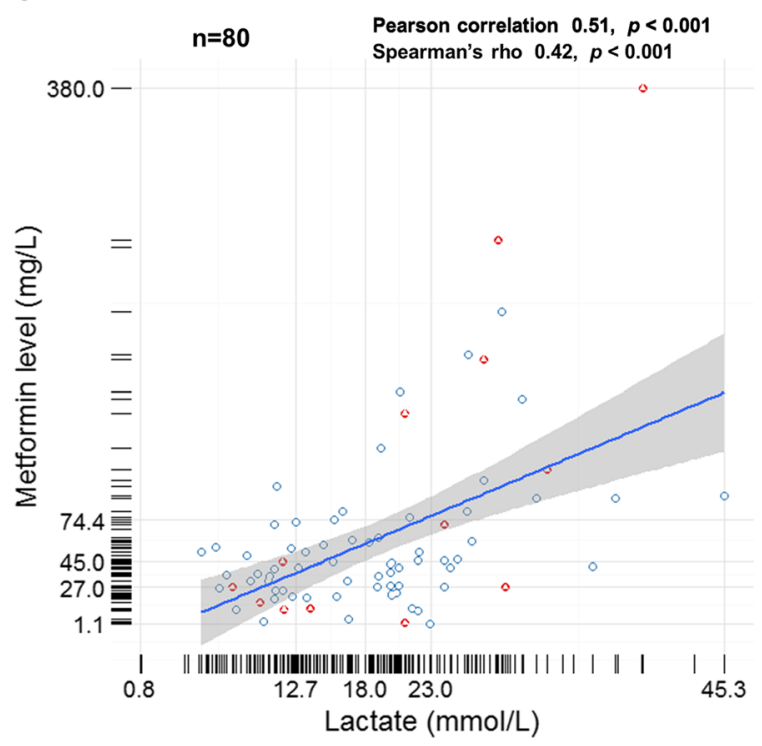

b

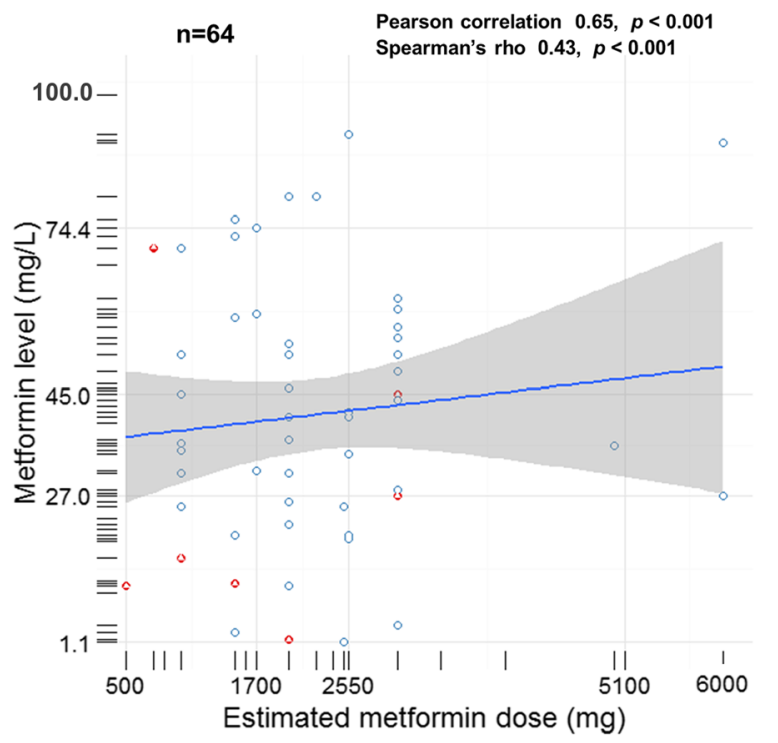

Alive $O$ Deceased

Fig. 2 Levels of serum metformin are plotted versus the serum lactate levels (a) and the estimated metformin dosage (b). There are significant positive correlation between serum metformin and lactate levels and between serum metformin levels and the estimated metformin dosage

about $50 \%$ based on single center experience [18]. We also found serum lactate level is a significant predictor for MALA-related mortality with a positive linear relationship particularly at greater than $20 \mathrm{mmol} / \mathrm{L}$. From a treatment planning perspective, renal replacement therapy (RRT) may play a critical role in reversing disturbances of lactic homeostasis but no single RRT modality shows superior performance than the other. Although our study contributes novel observations regarding MALA with kidney failure, the results need to be interpreted with caution due to publication bias, heterogeneity between studies, and moderate sample size.

The observed positive association between inappropriate dosing defined by conventional FDA serum creatinine criteria and mortality among MALA patients requiring RRT suggested the iatrogenic and preventable nature of MALA [12]. More interestingly, this association was inverted if the inappropriateness was defined by a recently proposed

Table 3 Odds ratios (ORs) with 95\% of confidence interval (Cl) of metformin-associated lactic acidosis (MALA)-related mortality for potentially predictive factors by univariable and multiple logistic regression

\begin{tabular}{|c|c|c|c|c|c|}
\hline Variable & Deceased/survivor (total) & $\begin{array}{l}\text { Crude analysis } \\
\text { OR }(95 \% \mathrm{Cl})\end{array}$ & $p$-value & $\begin{array}{l}\text { Multiple-adjustment } \\
\text { OR (95\% CI) } \\
\text { (deceased/survivor: } 23 / 152 \text {, total = 175) }\end{array}$ & $p$-value \\
\hline Blood lactate $(\mathrm{mmol} / \mathrm{L})$ & $36 / 198(234)$ & $1.08(1.03,1.13)$ & 0.001 & $1.09(1.02,1.17)$ & 0.009 \\
\hline Age (yr) & $40 / 212(252)$ & $0.99(0.97,1.01)$ & 0.297 & $1.03(0.98,1.08)$ & 0.236 \\
\hline Sex (ref: male) & $41 / 208(249)$ & $0.44(0.22,0.87)$ & 0.019 & $0.22(0.08,0.67)$ & 0.007 \\
\hline RRT modality & $41 / 210(251)$ & & & & \\
\hline IRRT & & Ref & - & Ref & - \\
\hline PIRRT & & $1.72(0.52,5.70)$ & 0.376 & $1.28(0.15,10.7)$ & 0.818 \\
\hline CRRT & & $2.36(1.01,5.52)$ & 0.048 & $3.13(0.81,12.1)$ & 0.098 \\
\hline PD & & $9.63(1.67,55.8)$ & 0.012 & $3.11(0.10,96.1)$ & 0.517 \\
\hline Metformin usage & $36 / 196(232)$ & & & & \\
\hline Suicidal (Ref: regular use) & & $2.19(1.02,4.70)$ & 0.044 & $0.80(0.15,4.23)$ & 0.790 \\
\hline Peak creatinine level (mg/dl) & 28/183 (211) & $0.80(0.70,0.91)$ & 0.001 & $0.77(0.65,0.91)$ & 0.003 \\
\hline
\end{tabular}

Abbreviations: CRRT continuous renal replacement therapy, IRRT intermittent renal replacement therapy, PD peritoneal dialysis, PIRRT prolonged intermittent renal replacement therapy, $R R T$ renal replacement therapy 


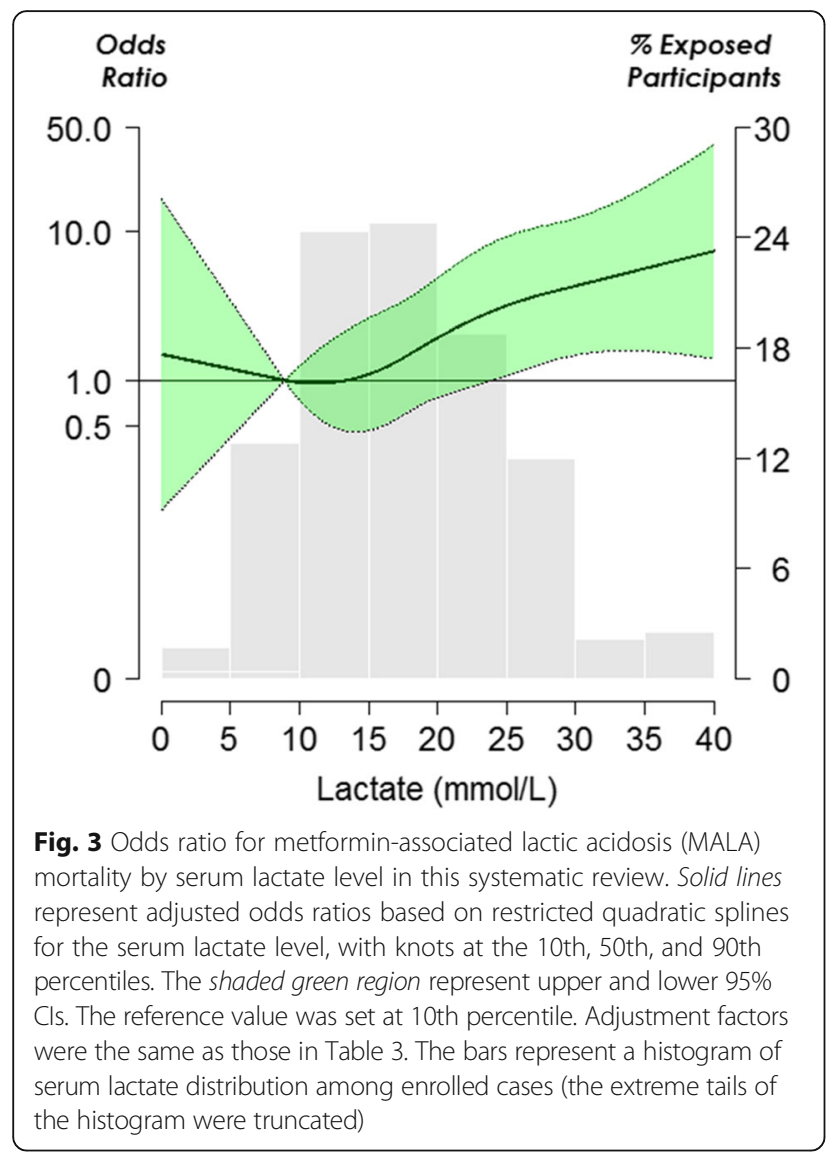

dosing recommendation (eGFR-dosing algorithm) [13]. Thus, the increasing sensitivity of alarming inappropriateness gained by applying the eGFR-dosing algorithm was of questionable clinical significance in the context of risk prevention. Furthermore, most cases with classification disagreement between the two different definitions were patients with CKD stage 3 . This finding indirectly supports that a cautious expansion of metformin use in patients with CKD stage 3 or 4 may be appropriate and the optimal dosing requires more evidence to justify its long term efficacy and safety profile among CKD population [9]. The drastic decline of kidney function among patients with appropriate metformin dose with concomitantly higher metformin and lactate concentration implied the primary etiologies of AKI played an important role in the development of MALA.

The traditional target of metformin is inhibition of mitochondrial complex-1, which subsequently impair the efficiency of mitochondrial oxidative phosphorylation and Adenosine triphosphate (ATP) production [19]. Mechanistic studies have linked metformin to activation of adenosine monophosphate (AMP)-activated protein kinase (AMPK) or inhibition of cyclic AMP formation following the drop of cellular energy charge [20, 21]. A recent study demonstrated that metformin inhibits mitochondrial glycerophosphate dehydrogenase (mGPD) causing an increased cytosolic redox state (increased cytosolic $\mathrm{NADH} / \mathrm{NAD}^{+}$ratio) that hinder the conversion of lactate to pyruvate by lactate dehydrogenase [22]. When serum metformin level increases abnormally due to either ingestion of a large amount of metformin and/ or to decreased kidney clearance of metformin, excessive production of lactate and proton will ensue.

Cumulating intracellular lactate is then transported into extracellular fluid (ECF) concomitantly with hydrogen 1:1 by proton-coupled monocarboxylate transporters (MCTs) [23]. To maintain acid-base homeostasis in ECF, proton reacts with bicarbonate to form water and carbon dioxide whereas lactate is metabolized by gluconeogenesis or oxidation after converting to pyruvate. Bicarbonate is then replenished by kidney via tubular reabsorption or net acid secretion $[24,25]$. This homeostasis is energy dependent and driven by decreased $\mathrm{NADH} / \mathrm{NAD}^{+}$ratio at cytosol, which is highly vulnerable in the context of MALA featuring with energy failure and increased intracellular redox status. As a result, the rapidly accumulating extracellular lactate and proton will consume equivalent amounts of bicarbonate in ECF until the maximum tolerable lactate threshold, around $20 \mathrm{mmol} / \mathrm{L}$ (Fig. 4b), is reached. Beyond this intrinsic threshold, the export of intracellular lactate/ proton is then severely limited, followed by irreversible cellular damage. However, among patients suffering from severe volume contraction and pre-existing metabolic alkalosis, the endogenous bicarbonate in ECF prior to the development MALA may raise this lactate threshold beyond $20 \mathrm{mmol} / \mathrm{L}$ [26]. To reverse the severe uncompensated acidosis, exogenous sodium bicarbonate infusion not only raises serum bicarbonate to neutralize proton acidity but also causes iatrogenic hypernatremia to create an extra "anion space" for excessive lactate in the price of systemic hyperosmolality (Fig. 4c). In this scenario, timely renal replacement therapy is the therapy of choice to remove excessive metformin and to correct intracellular acidosis while maintaining osmolar homeostasis as exemplified in a patient with MALA and an extremely high serum lactate level of $55 \mathrm{mmol} / \mathrm{L}$ successfully treated at our hospital (Additional file 1: Figure S4). This case highlighted the dynamics of how prescription of RRT was tailored to meet incredibly high metabolic demand.

Our findings are well consistent with a recent comprehensive review by Calello et al., in which a decision making algorithm for RRT in MALA was proposed [27]. The decision to initiate RRT is mainly triggered by serum lactate level and refractory severe acidosis. The proposed cut-off value of lactate, $20 \mathrm{mmol} / \mathrm{L}$, coincides with our dose-response analysis indicating a linear increase in mortality, particularly at lactate concentrations greater than $20 \mathrm{mmol} / \mathrm{L}$ [27]. The role of serum lactate in metforminrelated acidosis remains controversial from the diagnostic perspective as the elevation of lactate are usually induced from mutually reinforcing factors, for instance, predisposing 


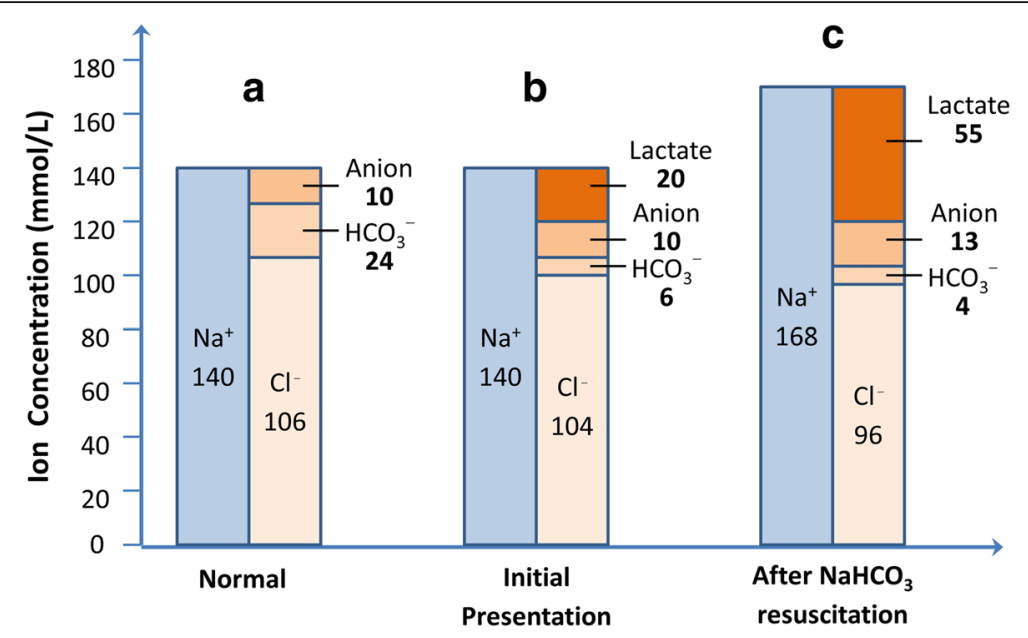

Fig. 4 The extracellular ionic compositional progression by Gamblegrams from our MALA case (The detailed information of the case was described in the Additional file 1: Figure S4). a normal physiology composition of major ions in extracellular fluid (ECF). b A 50-year-old woman case initially presented with severe lactic acidosis a few hours after suicidal metformin ingestion. Intracellular lactate was transported along with equivalent amount of protons into ECF via monocarboxylate transporters. Excess ECF protons were then buffered by bicarbonate and turned into $\mathrm{H}_{2} \mathrm{O}$ and $\mathrm{CO}_{2}$. Consequently, the increased amounts of lactate were approximately equivalent to the decreased consumption of bicarbonate. However, the failing kidney function could not replenish the consumed bicarbonate through renal ammoniagenesis or enhanced reabsorption, leading to increasingly severe acidosis. c To reverse acidosis, $622.5 \mathrm{mmol}$ of sodium bicarbonate was infused to neutralize protons moving out of the cell to ECF, along with lactate. The iatrogenic hypernatremia to $168 \mathrm{mmol} / \mathrm{L}$ created an extra anion space that allowed more lactate to move from intracellular fluid to ECF, resulting in more significant hyperlactatemia

impaired kidney function and resultant acidemia-related circulatory failure. But reversely, serum lactate could serve as a useful integrated marker to guide the initiation and adjustment of RRT. Compared to the general intensive care unit (ICU) population in which baseline serum lactate level is a reliable predictor of ICU mortality, the proposed lactate threshold for an increased mortality risk, $20 \mathrm{mmol} / \mathrm{L}$, is much higher than the usual alarming lactate range of 2.0$4.0 \mathrm{mmol} / \mathrm{L}$ in patient's lactemia mainly due to hypoperfusion (e.g., sepsis and trauma) [28]. Such discrepancy may reflect the unique pathogenesis of MALA - cellular energy failure and impaired redox state [21, 22]. More than half of the study population had hypothermia on arrival at the care hospital. This provides indirect evidence of cellular energy failure, as thermogenesis driven by ATP turnover would be compromised in MALA [29]. In the era of practicing individualized medicine, the bottom-line is that the modality of RRT should match to the patient's hemodynamic stability and the dosing of RRT has to properly correct acidosis and avoid iatrogenic hypernatremia, and should be adjusted to meet patient's metabolic demand (exemplified in Additional file 1: Figure S4). To address this current knowledge gap regarding the RRT prescription in MALA patients with severe acute kidney injury (e.g., Acute Kidney Injury Network (AKIN) stage 3) [16], an international consortium is needed to prospectively enroll patients worldwide using a standardized case report form to record detailed information about the metformin toxicokinetics and the three domains of RRT including mode, dose, and timing.

There were several interesting but unexpected findings in this study. First, a higher peak level of serum creatinine was associated with favorable survival. Indeed, apparent renal failure may help clinicians generate a high index of suspicion for MALA with appropriate evaluation resulting in earlier diagnosis and expedient delivery of RRT. On the other hand, patients with higher serum creatinine level at the initial presentation may also have a relatively indolent disease course rather than an acute episode of fulminant metabolic acidosis and circulatory collapse. However, our data was not sufficient to determine whether the timing of RRT was early or late as it is difficult, if not impossible, to define the onset of MALA. Second, female patients had a better survival than male patients. Although it is likely that the efficiency of RRT may be better among female patients due to smaller body size, the information of body mass index (BMI) was rarely available in the published reports. Future prospective research is warranted to clarify the sex difference or the effect of BMI.

\section{Limitation}

Although the comprehensive nature of this systematic review can be interpreted as strength, several limitations deserve particular mention in the current work. Publication bias is a critical concern in this study as severe cases with unexpected favorable outcomes may be more likely 
reported by clinicians and accepted by medical journals. We compared the mortality rate of selected MALA patients between 1995 and 2010 with those from a pharmacovigilence database covering the same time span and the results were not too different $(17.7 \%$ vs. $25 \%)$ [30]. The much lower mortality in our study population compared to the historical series may reflect the efficient support role of the RRT in maintaining the acid-base homeostasis in MALA [18, 31]. On the other hand, misclassification of the diagnosis of MALA can not be completely excluded. However, as all enrolled reports were peer-reviewed, misdiagnosis of MALA may be minimized. Residual confounding, especially unknown or unmeasured confounding, is another serious concern in this study. For example, it is not possible to standardize the onset and severity of MALA among the retrospectively enrolled cases to determine whether the intervention of RRT is early or late. Subsequently, the conclusions drawn from this study regarding the determinants of mortality are very limited to infer causality and generalizability [32]. However, this real-world issue may not be completely avoided by prospective studies. Considering the rarity and severity of MALA, systematically analyzing case reports in the literature may be a realistic approach to describe the practice pattern and trend of RRT use in MALA.

\section{Conclusions}

Our study suggests that predialysis level of serum lactate level is an important marker of mortality in MALA patients requiring RRT with a linear dose-response relationship. There is no differential survival advantage among RRT modalities and significant knowledge gaps remain regarding the optimal timing, mode, and dose of RRT. Standardization of MALA case report form would be the fundamental step toward guideline development.

\section{Additional file}

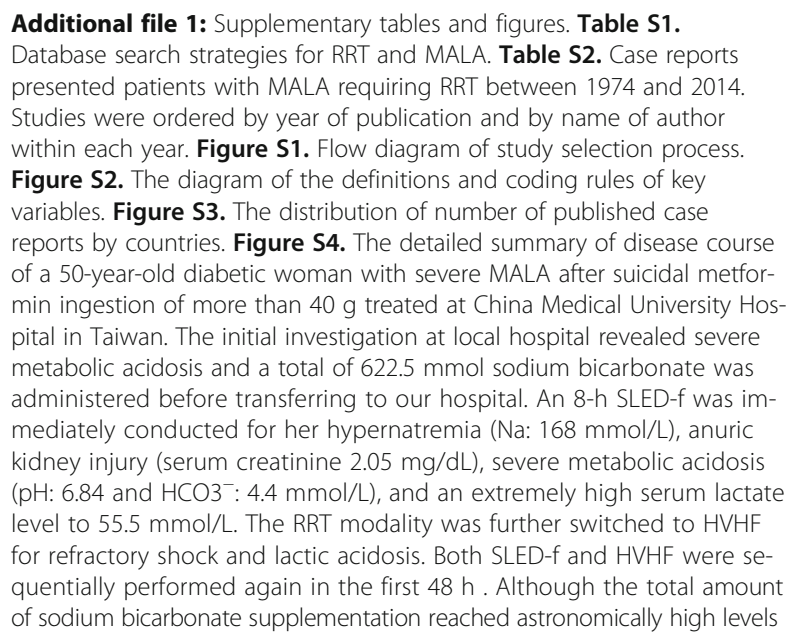

(1660 mmol via intravenous infusion; $4400 \mathrm{mmol}$ via replacement fluid), the serum sodium concentration could be maintained at normal range within $24 \mathrm{~h}$ of RRT initiation. The RRT modalities were shifted to $\mathrm{CWH}$ and then IHD as condition stabilized, and the patient obtained independence from RRT after 20 days of treatment. Her peak serum lactate level of $55.5 \mathrm{mmol} / \mathrm{l}$ is more than any case we have reviewed in this systematic review. Abbreviations: Bicarb, each yellow rectangle of "Bicarb" stands for $250 \mathrm{ml}$ of $7 \%$ sodium bicarbonate; BT, body temperature; $\mathrm{CWH}$, continuous venovenous hemofiltration (replacement flow $=35 \mathrm{ml} / \mathrm{kg} / \mathrm{h}$ ); HR, heart rate; HVHF, high volume hemofiltration (replacement flow $=70 \mathrm{ml} / \mathrm{kg} / \mathrm{h}$ ); IE, inotropic equivalents; SBP, systolic blood pressure; SLED-f, sustained low-efficiency hemodiafiltration. (DOCX $768 \mathrm{~kb})$

\section{Abbreviations}

ACEls: Angiotensin-converting-enzyme inhibitors; AKl: Acute kidney injury; AMP: Adenosine monophosphate; AMPK: Adenosine monophosphateactivated protein kinase; ARBs: Angiotensin II receptor blockers; ATP: Adenosine triphosphate; CRRT: Continuous renal replacement therapy; $\mathrm{CWH}$ : Continuous venovenous hemofiltration; CWHD: Continuous venovenous hemodialysis; CWHDF: Continuous venovenous hemodiafiltration; ECF: Extracellular fluid; ESRD: End stage renal disease; FDA: Food and Drug Administration; HF: Hemofiltration; HVHF: High volume hemofiltration; IHD: Intermittent hemodialysis; IRRT: Intermittent renal replacement therapy; MALA: Metformin-associated lactic acidosis; MCTs: Monocarboxylate transporters; mGPD: Mitochondrial glycerophosphate dehydrogenase; NAD: Nicotinamide adenine dinucleotide;

NSAID: Nonsteroidal anti-inflammatory drugs; PD: Peritoneal dialysis; PIRRT: Prolonged intermittent renal replacement therapy; RRT: Renal replacement therapy

\section{Acknowledgements}

Not applicable.

\section{Funding}

This study was supported by physician scientist training program from the China Medical University Hospital.

\section{Availability of data and materials}

All data generated or analyzed during this study are included in this published article and its supplementary information files.

\section{Authors' contributions}

CCK, IWT, and HCY prepared research data, conducted statistical analysis, and manuscript writing. CWT and JYW participated in biochemical and physiology counseling and conducted manuscript revision and editing. All authors read and approved the final manuscript.

\section{Ethics approval and consent to participate} Not applicable.

\section{Consent for publication}

Written informed consent was obtained from the patient for publication of the Additional file 1: Figure S4.

\section{Competing interests}

All authors declared no competing interests.

\section{Publisher's Note}

Springer Nature remains neutral with regard to jurisdictional claims in published maps and institutional affiliations.

\section{Author details}

${ }^{1}$ Kidney Institute and Division of Nephrology, Department of Internal Medicine, China Medical University Hospital and College of Medicine, China Medical University, 2, Yude Rd., North Dist, Taichung City 404, Taiwan. ${ }^{2}$ Big Data Center, China Medical University Hospital and College of Medicine, China Medical University, Taichung, Taiwan. ${ }^{3}$ Department of Internal Medicine, National Taiwan University Hospital Yun-Lin Branch, Yun-Lin, Taiwan. 
Received: 30 December 2016 Accepted: 26 June 2017 Published online: 10 July 2017

\section{References}

1. American Diabetes A. Standards of medical care in diabetes-2014. Diabetes Care. 2014;37(Suppl 1):S14-80.

2. NICE. Type 2 diabetes: the management of type 2 diabetes. In. London: National Institute for Health and Care Excellence; 2009.

3. Orchard TJ, Temprosa M, Goldberg R, Haffner S, Ratner R, Marcovina S, Fowler S, Diabetes Prevention Program Research G. The effect of metformin and intensive lifestyle intervention on the metabolic syndrome: the Diabetes Prevention Program randomized trial. Ann Intern Med. 2005;142(8):611-9.

4. Ehrmann DA. Polycystic ovary syndrome. N Engl J Med. 2005;352(12):1223-36.

5. Kajbaf F, Lalau JD. The prognostic value of blood $\mathrm{pH}$ and lactate and metformin concentrations in severe metformin-associated lactic acidosis. BMC Pharmacol Toxicol. 2013;14:22

6. Richy FF, Sabido-Espin M, Guedes S, Corvino FA, Gottwald-Hostalek U. Incidence of lactic acidosis in patients with type 2 diabetes with and without renal impairment treated with metformin: a retrospective cohort study. Diabetes Care. 2014;37(8):2291-5.

7. Lalau JD. Lactic acidosis induced by metformin: incidence, management and prevention. Drug Saf. 2010;33(9):727-40.

8. Bailey CJ, Turner RC. Metformin. N Engl J Med. 1996;334(9):574-9.

9. Kuo CC, Yeh HC, Chen B, Tsai CW, Lin YS, Huang CC. Prevalence of metformin use and the associated risk of metabolic acidosis in US diabetic adults with CKD: a National Cross-Sectional Study. Medicine. 2015:94(51):e2175.

10. Moher D, Liberati A, Tetzlaff J, Altman DG, Group P. Preferred reporting items for systematic reviews and meta-analyses: the PRISMA statement. Ann Intern Med. 2009;151(4):264-9. W264

11. Luft D, Deichsel G, Schmulling RM, Stein W, Eggstein M. Definition of clinically relevant lactic acidosis in patients with internal diseases. Am J Clin Pathol. 1983;80(4):484-9.

12. U.S. Food and Drug Administration (FDA): Glucophage (metformin hydrochloride tablets) 2008.

13. Inzucchi SE, Lipska KJ, Mayo H, Bailey CJ, McGuire DK. Metformin in patients with type 2 diabetes and kidney disease: a systematic review. JAMA. 2014; 312(24):2668-75

14. Levey AS, Stevens LA, Schmid CH, Zhang YL, Castro AF 3rd, Feldman HI, Kusek JW, Eggers P, Van Lente F, Greene T, et al. A new equation to estimate glomerular filtration rate. Ann Intern Med. 2009;150(9):604-12.

15. Levey AS, Eckardt KU, Tsukamoto Y, Levin A, Coresh J, Rossert J, De Zeeuw D, Hostetter TH, Lameire N, Eknoyan G. Definition and classification of chronic kidney disease: a position statement from Kidney Disease: Improving Global Outcomes (KDIGO). Kidney Int. 2005;67(6):2089-100.

16. Mehta RL, Kellum JA, Shah SV, Molitoris BA, Ronco C, Warnock DG, Levin A, Acute Kidney Injury N. Acute kidney injury network: report of an initiative to improve outcomes in acute kidney injury. Crit Care. 2007;11(2):R31.

17. Gesmann M, de Castillo D. Using the Google visualisation API with R. R J. 2011;3:40-4.

18. Seidowsky A, Nseir S, Houdret N, Fourrier F. Metformin-associated lactic acidosis: a prognostic and therapeutic study. Crit Care Med. 2009;37(7):2191-6.

19. Andrzejewski S, Gravel SP, Pollak M, St-Pierre J. Metformin directly acts on mitochondria to alter cellular bioenergetics. Cancer Metab. 2014;2:12.

20. Viollet B, Guigas B, Sanz Garcia N, Leclerc J, Foretz M, Andreelli F. Cellular and molecular mechanisms of metformin: an overview. Clin Sci. 2012;122(6):253-70.

21. Foretz M, Guigas B, Bertrand L, Pollak M, Viollet B. Metformin: from mechanisms of action to therapies. Cell Metab. 2014;20(6):953-66.

22. Madiraju AK, Erion DM, Rahimi Y, Zhang XM, Braddock DT, Albright RA, Prigaro BJ, Wood JL, Bhanot S, MacDonald MJ, et al. Metformin suppresses gluconeogenesis by inhibiting mitochondrial glycerophosphate dehydrogenase. Nature. 2014;510(7506):542-6.

23. Klier M, Andes FT, Deitmer JW, Becker HM. Intracellular and extracellular carbonic anhydrases cooperate non-enzymatically to enhance activity of monocarboxylate transporters. J Biol Chem. 2014;289(5):2765-75.

24. Abu Hossain S, Chaudhry FA, Zahedi K, Siddiqui F, Amlal H. Cellular and molecular basis of increased ammoniagenesis in potassium deprivation. Am J Physiol Renal Physiol. 2011;301(5):F969-78.

25. Jacobs RA, Meinild AK, Nordsborg NB, Lundby C. Lactate oxidation in human skeletal muscle mitochondria. Am J Phys Endocrinol Metab. 2013;304(7):E686-94.

26. Kraut JA, Madias NE. Lactic acidosis. N Engl J Med. 2014;371(24):2309-19.
27. Calello DP, Liu KD, Wiegand TJ, Roberts DM, Lavergne V, Gosselin S, Hoffman RS, Nolin TD, Ghannoum M, Extracorporeal treatments in Poisoning W. Extracorporeal treatment for Metformin poisoning: systematic review and recommendations from the extracorporeal treatments in Poisoning Workgroup. Crit Care Med. 2015;43(8):1716-30.

28. Kruse O, Grunnet N, Barfod C. Blood lactate as a predictor for in-hospital mortality in patients admitted acutely to hospital: a systematic review. Scand J Trauma Resusc Emerg Med. 2011;19:74.

29. Silva JE. Thermogenic mechanisms and their hormonal regulation. Physiol Rev. 2006;86(2):435-64.

30. Kajbaf F, Lalau JD. Mortality rate in so-called "metformin-associated lactic acidosis": a review of the data since the 1960s. Pharmacoepidemiol Drug Saf. 2014;23(11):1123-7.

31. Peters N, Jay N, Barraud D, Cravoisy A, Nace L, Bollaert PE, Gibot S. Metforminassociated lactic acidosis in an intensive care unit. Crit Care. 2008;12(6):R149.

32. Albrecht J, Werth VP, Bigby M. The role of case reports in evidence-based practice, with suggestions for improving their reporting. J Am Acad Dermatol. 2009;60(3):412-8.

\section{Submit your next manuscript to BioMed Central and we will help you at every step:}

- We accept pre-submission inquiries

- Our selector tool helps you to find the most relevant journal

- We provide round the clock customer support

- Convenient online submission

- Thorough peer review

- Inclusion in PubMed and all major indexing services

- Maximum visibility for your research

Submit your manuscript at www.biomedcentral.com/submit
C) Biomed Central 